\title{
RPC upgrade project for CMS Phase II
}

To cite this article: M.I. Pedraza-Morales et al 2020 JINST 15 C05072

View the article online for updates and enhancements.

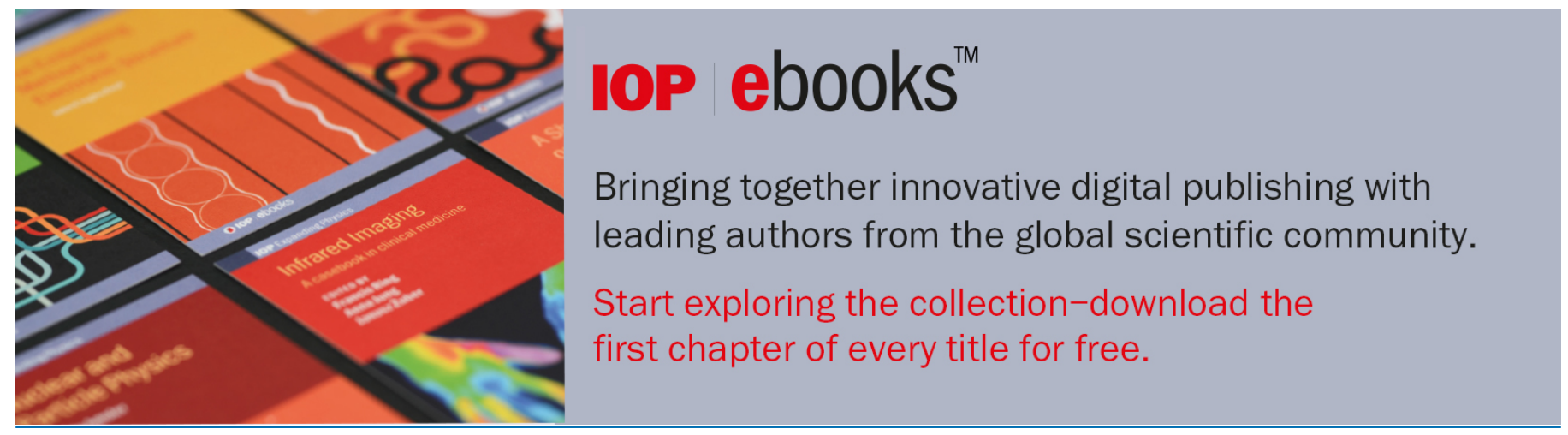


$144^{\text {Th }}$ Workshop on Resistive Plate Chambers and Related Detectors

19-23 FEBRUARY, 2018

Puerto Vallarta, Jalisco state, Mexico

\title{
RPC upgrade project for CMS Phase II
}

\author{
M.I. Pedraza-Morales, ${ }^{v, 1}$ A. Fagot, ${ }^{a}$ M. Gul, ${ }^{a}$ C. Roskas, ${ }^{a}$ M. Tytgat, ${ }^{a}$ N. Zaganidis, ${ }^{a}$ \\ S. Fonseca De Souza, ${ }^{b}$ A. Santoro, ${ }^{b}$ F. Torres Da Silva De Araujo, ${ }^{b}$ A. Aleksandrov, ${ }^{c}$ \\ R. Hadjiiska, ${ }^{c}$ P. laydjiev, ${ }^{c}$ M. Rodozov, ${ }^{c}$ M. Shopova, ${ }^{c}$ G. Sultanov, ${ }^{c}$ A. Dimitrov, ${ }^{d}$ L. Litov, ${ }^{d}$ \\ B. Pavlov, ${ }^{d}$ P. Petkov, ${ }^{d}$ A. Petrov, ${ }^{d}$ S.J. Qian, ${ }^{e}$ D. Han, ${ }^{f}$ W. Yi, ${ }^{f}$ C. Avila, ${ }^{g}$ A. Cabrera, ${ }^{g}$ \\ C. Carrillo, ${ }^{g}$ M. Segura, ${ }^{g}$ S. Aly, ${ }^{h}$ Y. Assran,,${ }^{h}$ A. Mahrous, ${ }^{h}$ A. Mohamed, ${ }^{h}$ C. Combaret,${ }^{i}$ \\ M. Gouzevitch, ${ }^{i}$ G. Grenier, ${ }^{i}$ F. Lagarde,${ }^{i}$ I.B. Laktineh, ${ }^{i}$ H. Mathez,${ }^{i}$ L. Mirabito, ${ }^{i}$ \\ K. Shchablo, ${ }^{i}$ I. Bagaturia, ${ }^{j}$ D. Lomidze,${ }^{j}$ I. Lomidze, ${ }^{j}$ L.M. Pant, ${ }^{k}$ V. Bhatnagar, ${ }^{l}$ R. Gupta, ${ }^{l}$ \\ R. Kumari, ${ }^{l}$ M. Lohan, ${ }^{l}$ J.B. Singh, ${ }^{l}$ V. Amoozegar, ${ }^{m}$ B. Boghrati, ${ }^{m, n}$ H. Ghasemy, ${ }^{m}$ \\ S. Malmir ${ }^{m}$ M. Mohammadi Najafabadi, ${ }^{m}$ M. Abbrescia, ${ }^{o}$ A. Gelmi, ${ }^{o}$ G. laselli, ${ }^{o}$ S. Lezki, ${ }^{o}$ \\ G. Pugliese, ${ }^{o}$ L. Benussi, ${ }^{p}$ S. Bianco, ${ }^{p}$ D. Piccolo,${ }^{p}$ F. Primavera, ${ }^{p}$ S. Buontempo, ${ }^{q}$ \\ A. Crescenzo, ${ }^{q}$ G. Galati, ${ }^{q}$ F. Fienaga, ${ }^{q}$ I. Orso, ${ }^{q}$ L. Lista, ${ }^{q}$ S. Meola, ${ }^{q}$ P. Paolucci, ${ }^{q}$ \\ E. Voevodina, ${ }^{q}$ A. Braghieri, ${ }^{r}$ P. Montagna, ${ }^{r}$ M. Ressegotti, ${ }^{r}$ C. Riccardi, ${ }^{r}$ P. Salvini, ${ }^{r}$ \\ P. Vitulo, ${ }^{r}$ S.W. Cho, ${ }^{s}$ S.Y. Choi, ${ }^{s}$ B. Hong,${ }^{s}$ K.S. Lee,${ }^{s}$ J.H. Lim, ${ }^{s}$ S.K. Park, ${ }^{s}$ J. Goh, ${ }^{t, a}$ \\ T.J. Kim, ${ }^{t}$ S. Carrillo Moreno, ${ }^{u}$ O. Miguel Colin, ${ }^{u}$ F. Vazquez Valencia, ${ }^{u}$ \\ S. Carpinteyro Bernardino, ${ }^{v}$ J. Eysermans, ${ }^{v}$ C. Uribe Estrada, ${ }^{v}$ R. Reyes-Almanza, ${ }^{w}$ \\ M.C. Duran-Osuna, ${ }^{w}$ G. Ramirez-Sanchez, ${ }^{w}$ A. Sanchez-Hernandez, ${ }^{w}$ R.I. Rabadan-Trejo, ${ }^{w}$ \\ H. Castilla-Valdez, ${ }^{w}$ A. Radi, ${ }^{x}$ H. Hoorani, ${ }^{y}$ S. Muhammad, ${ }^{y}$ M.A. Shah ${ }^{y}$ and I. Crotty ${ }^{z}$ \\ ${ }^{a}$ Ghent university, Department of Physics and Astronomy, Proeftuinstraat 86, B-9000 Ghent, Belgium \\ ${ }^{b}$ Departamento de Fisica Nuclear e Altas Energias, Instituto de Fisica, \\ Universidade do Estado do Rio de Janeiro, \\ Rua Sao Francisco Xavier, 524, BR - Rio de Janeiro 20559-900, RJ, Brazil \\ ${ }^{c}$ Bulgarian Academy of Sciences, Institute for Nuclear Research and Nuclear Energy, \\ Tzarigradsko shaussee Boulevard 72, BG-1784 Sofia, Bulgaria \\ ${ }^{d}$ Faculty of Physics, University of Sofia, 5 James Bourchier Boulevard, BG-1164 Sofia, Bulgaria \\ ${ }^{e}$ School of Physics, Peking University, Beijing 100871, China \\ ${ }^{f}$ Tsinghua University, Shuangqing Rd, Haidian Qu, Beijing, China \\ ${ }^{g}$ Universidad de Los Andes, Apartado Aereo 4976, Carrera 1E, no. 18A 10, CO-Bogota, Colombia
}

${ }^{1}$ Corresponding author. 
${ }^{h}$ Egyptian Network for High Energy Physics, Academy of Scientific Research and Technology,

101 Kasr El-Einy St. Cairo Egypt

${ }^{i}$ Universite de Lyon, Universite Claude Bernard Lyon 1, CNRS-IN2P3,

Institut de Physique Nucleaire de Lyon, Villeurbanne, France

${ }^{j}$ Georgian Technical University, 77 Kostava Str., Tbilisi 0175, Georgia

${ }^{k}$ Nuclear Physics Division, Bhabha Atomic Research Centre, Mumbai 400 085, India

${ }^{l}$ Department of Physics, Panjab University, Chandigarh Mandir 160 014, India

${ }^{m}$ School of Particles and Accelerators, Institute for Research in Fundamental Sciences (IPM), Tehran, Iran

${ }^{n}$ School of Engineering, Damghan University, Damghan, Iran

${ }^{o}$ INFN, Sezione di Bari, Via Orabona 4, IT-70126 Bari, Italy

${ }^{p}$ INFN, Laboratori Nazionali di Frascati (LNF), Via Enrico Fermi 40, IT-00044 Frascati, Italy

${ }^{q}$ INFN, Sezione di Napoli, Complesso Univ. Monte S. Angelo, Via Cintia, IT-80126 Napoli, Italy

${ }^{r}$ INFN, Sezione di Pavia, Via Bassi 6, IT-Pavia, Italy

${ }^{s}$ Department of Physics, Korea University, 145 Anam-ro, Seongbuk-gu, Seoul 02841, Republic of Korea

${ }^{t}$ Hanyang University, 222 Wangsimni-ro, Sageun-dong, Seongdong-gu, Seoul, Republic of Korea

${ }^{t t}$ Kyunghee University, 26 Kyungheedae-ro, Hoegi-dong, Dongdaemun-gu, Seoul, Republic of Korea

${ }^{u}$ Universidad Iberoamericana, Mexico City, Mexico

${ }^{v}$ Benemerita Universidad Autonoma de Puebla, Puebla, Mexico

${ }^{w}$ Cinvestav, Av. Instituto Politécnico Nacional No. 2508,

Colonia San Pedro Zacatenco, CP 07360, Ciudad de Mexico D.F., Mexico

${ }^{x}$ Sultan Qaboos University, Al Khoudh,Muscat 123, Oman

${ }^{y}$ National Centre for Physics, Quaid-i-Azam University, Islamabad, Pakistan

${ }^{z}$ Department of Physics, Wisconsin University, Madison, WI 53706, U.S.A.

E-mail: mpedraza@cern.ch

AвstRact: The Muon Upgrade Phase II of the Compact Muon Solenoid (CMS) aims to guarantee the optimal conditions of the present system and extend the $\eta$ coverage to ensure a reliable system for the High Luminosity Large Hadron Collider (HL-LHC) period. The Resistive Plate Chambers (RPCs) system will upgrade the off-detector electronics (called link system) of the chambers currently installed chambers and place improved RPCs (iRPCs) to cover the high pseudo-rapidity region, a challenging region for muon reconstruction in terms of background and momentum resolution. In order to find the best option for the iRPCs, an R\&D program for new detectors was performed and real size prototypes have been tested in the Gamma Irradiation Facility (GIF++) at CERN. The results indicated that the technology suitable for the high background conditions is based on High Pressure Laminate (HPL) double-gap RPC. The RPC Upgrade Phase II program is planned to be ready after the Long Shutdown 3 (LS3).

Keywords: Resistive-plate chambers; Gaseous detectors; Particle tracking detectors (Gaseous detectors)

ARXIV EPrint: 1806.11503 


\section{Contents}

1 Muon spectrometer 1

2 RPC Muon Upgrade Project 1

$3 \quad \eta$ coverage extension 2

3.1 Muon identification efficiency 2

3.2 Improved RPCs 3

4 Sustainability of the current system 5

4.1 Link Board upgrade for the current RPC system 5

$\begin{array}{lll}4.2 & \text { Eco-gas studies } & 6\end{array}$

5 Summary 7

\section{Muon spectrometer}

The muon system, described in detail in [1], uses three different technologies; drift tubes (DT) in the barrel region, cathode strip chambers (CSC) in the end-cap region, and resistive plate chambers (RPC) in both the barrel and end-cap. It covers a pseudo-rapidity region $|\eta|<2.4$. The DTs and RPCs in the barrel cover the $\eta$ region $|\eta|<1.2$, the CSCs and the RPCs in the end-caps cover the $\eta$ region $0.9<|\eta|<1.8$, while the CSCs cover up to $|\eta|<2.4$. A schematic view of the CMS detector with all Phase-2 muon upgrades is shown in figure 1, where the location of the muon chambers is highlighted. The current RPC system covers $|\eta|<1.8$. The barrel region is divided into 5 separate wheels, while the end-caps in 4 disks both in the forward and backward directions, with the 4th disk installed during the first LHC long shutdown in 2013-2014 (LS1). In total, there are 1056 RPC chambers, covering an area of about $3950 \mathrm{~m}^{2}$, equipped with about 137,000 readout strips [7].

\section{RPC Muon Upgrade Project}

The LHC has prepared and approved a major upgrade of the accelerators to increase the sensitivity for new physics searches and high precision measurements, the so-called High Luminosity Large Hadron Collider (HL-LHC) period. The instantaneous luminosity will reach a maximum of $7.5 \times 10^{34} \mathrm{~cm}^{-2} \mathrm{~s}^{-1}$, more than seven times the nominal one, expecting 200 collisions per bunch crossing to collect up to $4000 \mathrm{fb}^{-1}$ by the end of the LHC operations on 2039 [2]. The RPC Muon Upgrade Project consists of the extension of the RPCs system to the very forward region and the sustainability of the current system to ensure a reliable redundancy system for the HL-LHC. 


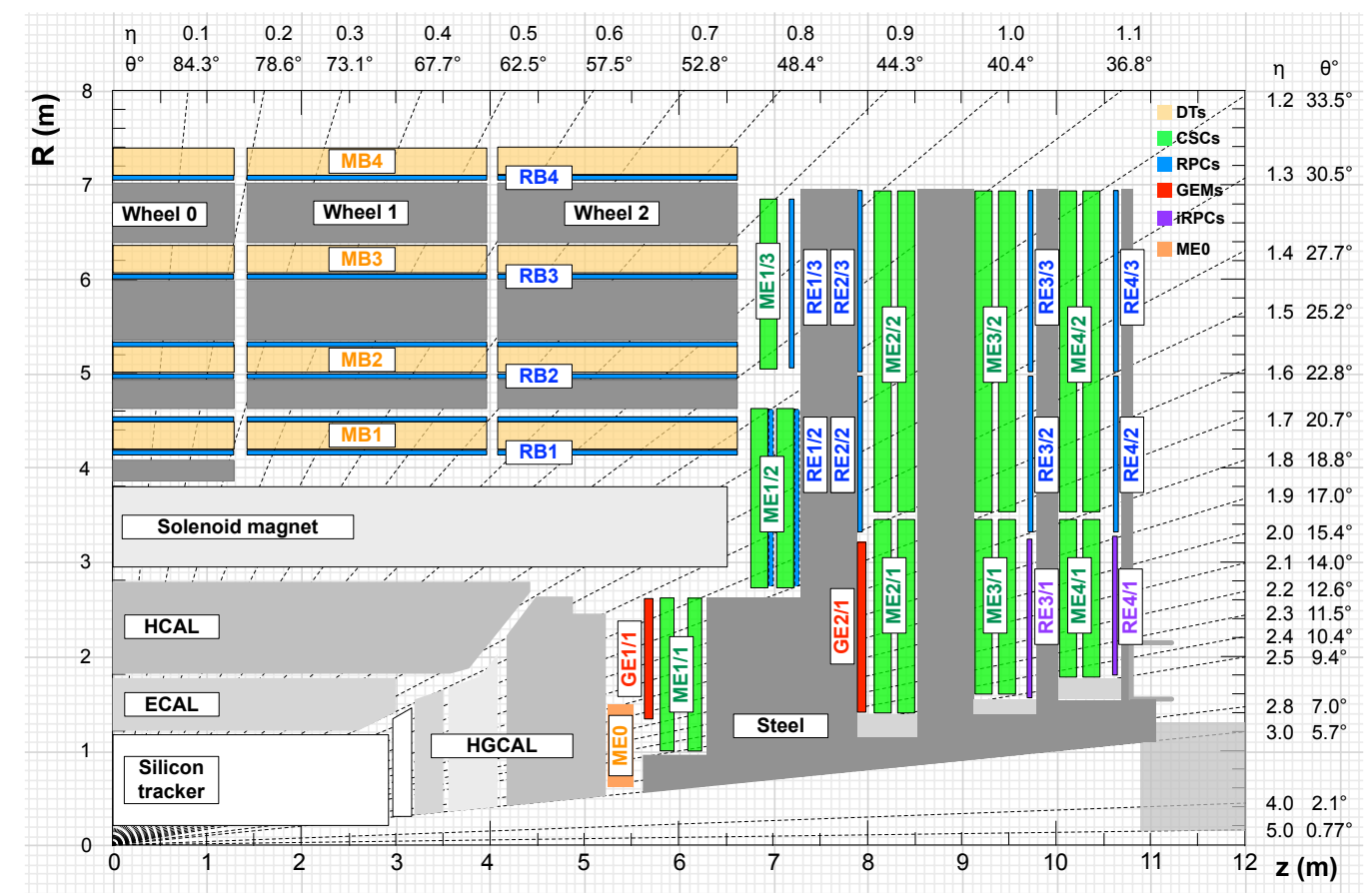

Figure 1. An R-z cross section of a quadrant of the CMS detector, including the Phase-2 upgrades (RE3/1, RE4/1, GE1/1, GE2/1, ME0). The interaction point is at the lower left corner. The acronym iRPCs in the legend refers to the new iRPC chambers RE3/1 and RE4/1. The locations of the various muon stations are shown in color (MB for DTs, ME for CSC, RB and RE for RPC, GE and ME0 for GEM (Gas Electron Multiplier)). M denotes Muon, B stands for Barrel and E for End-cap. The magnet yoke is represented by the dark gray areas.

\section{$3 \quad \eta$ coverage extension}

During the HL-LHC the background induced by neutrons, photons, electrons, positrons and a large number of particles crossing the CMS detector quasi simultaneously, will reach $700 \mathrm{~Hz} / \mathrm{cm}^{2}$ in the hottest points of the region of interest, for an instantaneous luminosity $5 \times 10^{34} \mathrm{~cm}^{-2} \mathrm{~s}^{-1}$, figure 2 shows the expected hit rate. This makes the association of individual hits to tracks and thus muon triggering, identification, momentum and charge measurements a major challenge. To increase the number of hits recorded for a single particle and then improve the muon reconstruction in this difficult zone, the $\eta$ coverage extension is proposed to go from $|\eta|=1.8$ to $|\eta|=2.4$. That means adding chambers in the innermost part of the two most distant end-caps of the muon spectrometer, $\mathrm{RE} 3 / 1$, and RE4/1.

\subsection{Muon identification efficiency}

The addition of RE3/1 and RE4/1 will increase the number of hits per muon track. Even though the CSCs system covers the region, there are steep drops in efficiency in some regions due to the geometry of the system. Including the RPC information into the trigger primitive will mitigate this effect, resulting in an overall increase in efficiency. Figure 3 shows the efficiency of finding trigger primitive stubs at the level of station 3 and station 4, with and without the addition of the current RPC and new iRPC information. 


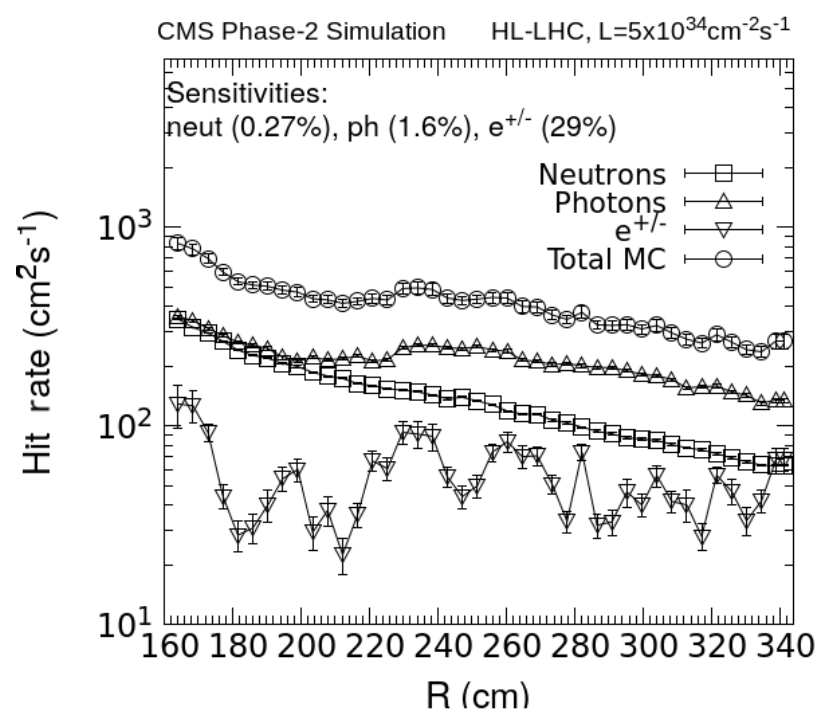

Figure 2. Expected hit rate due to neutrons, photons, electrons and positrons at the HL-LHC in the RE3/1 chambers. In the upper part of the figure the sensitivities used in the simulation for each particle are also reported. The expected hit rates in RE4/1 are similar.
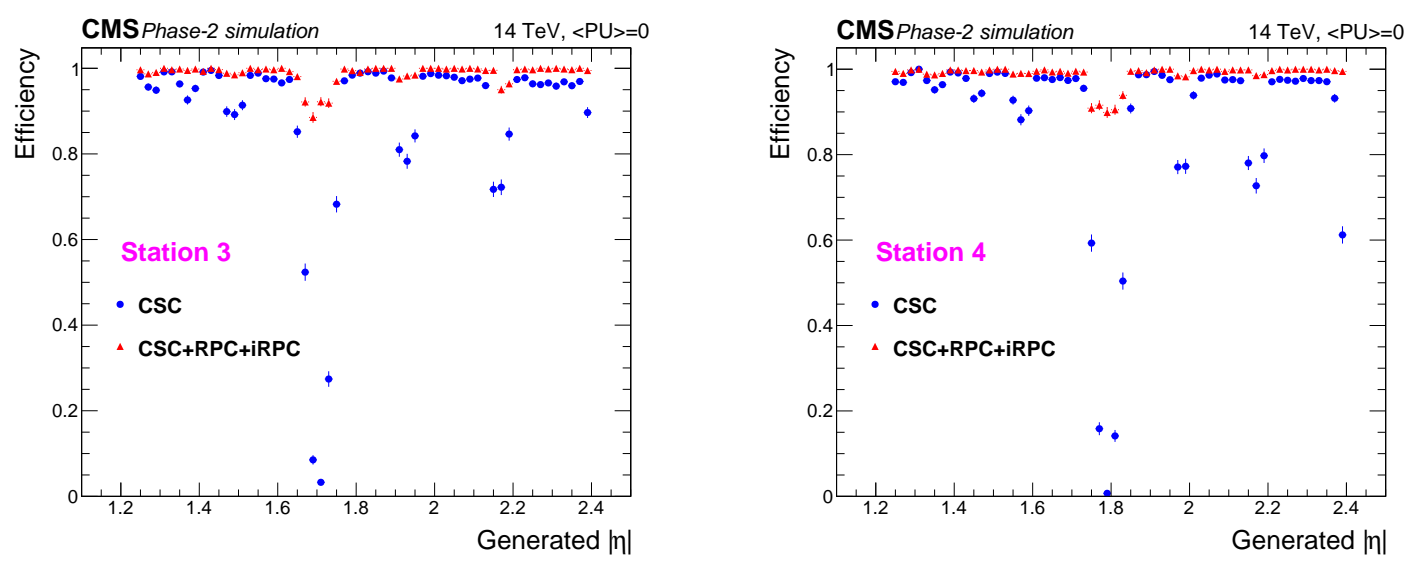

Figure 3. Impact of RPC hit inclusion on the local trigger primitive efficiency in station 3 (left) and station 4 (right). The contribution of iRPC starts above $|\eta|=1.8$.

\subsection{Improved RPCs}

We have applied a safety factor of three to the hit rate and required that the new chambers stand $2 \mathrm{kHz} / \mathrm{cm}^{2}$ of gamma radiation while maintaining a hit efficiency above $95 \%$. A double-gap RPC with reduced High Pressure Laminate (HPL) electrode and reduced gas gap thickness, with respect to the present system, has been proposed. These modifications will allow the iRPCs to satisfy the space requirements of the experiment. In addition to satisfy the required performance in rate capability and performance the latest available technology is being used for the design of the Front End Board (FEB) capable of handling the increased background in the forward innermost region. A separate paper has been prepared for this conference. 
The pickup charge was studied in six double-gap RPCs with gap thickness between 1.0 and $2.0 \mathrm{~mm}[4]$ at the KOrean DEtector Laboratory (KODEL). The gaps were equipped with a voltage sensitive front-end board with a charge threshold of about $50 \mathrm{fC}$, which is lower than the value of the present RPC system front-end electronics by about a factor of three. Figure 4 shows the induced charges drawn in double-gap RPCs for the different gas gap as a function of the effective electric field strength. Electric field values are converted to the effective ones at the reference values of pressure, $\mathrm{P}=990 \mathrm{hPa}$, and temperature, $\mathrm{T}=293 \mathrm{~K}$.

This implies that thinner gaps will preserve the operational plateau when the digitization threshold is lowered to increase the detection sensitivity [5]. Taking into account that thinner gaps would be more sensitive to non-uniformities, the value $1.4 \mathrm{~mm}$ was taken, for the baseline design of the iRPCs, as a safe compromise.

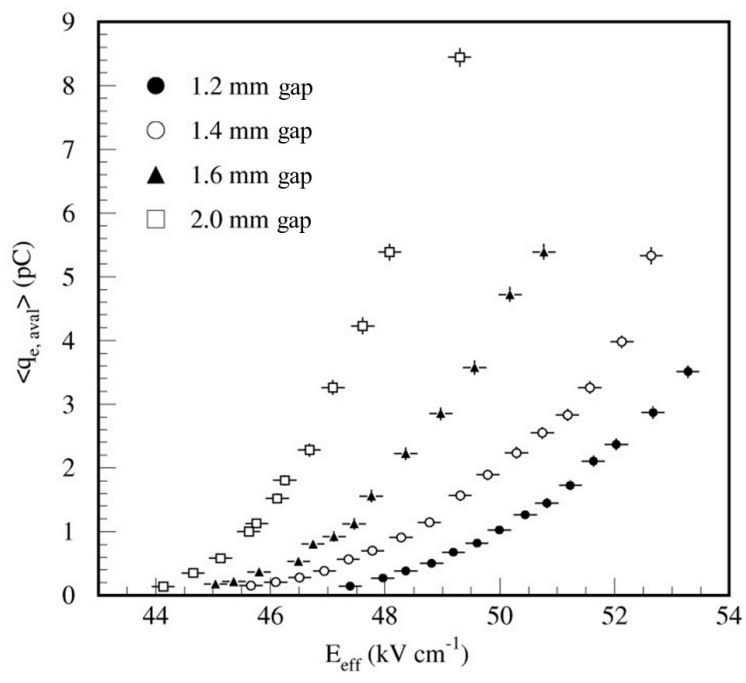

Figure 4. Average charge per avalanche measured on $1.20 \mathrm{~mm}$ (full circles), $1.40 \mathrm{~mm}$ (open circles), $1.60 \mathrm{~mm}$ (triangles), and $2.0 \mathrm{~mm}$ (squares) double-gap RPCs, as a function of the effective electric field strength.

In order to validate the performance of the baseline iRPC under different background conditions, a large size prototype was tested at the Gamma Irradiation Facility (GIF++) with muon beam. The prototype was a trapezoidal chamber (long base $92 \mathrm{~cm}$, short base $63 \mathrm{cms}$, and height $166.3 \mathrm{~cm}$ ) with $2 \mathrm{~cm}$ wide strips and the same front-end electronics threshold used for the tests at KODEL. Figure 5 shows the rate capability of the prototype with more than $95 \%$ efficiency at $\sim 2 \mathrm{kHz} / \mathrm{cm}^{2}$, where the working voltage shift for the highest rate is less than $300 \mathrm{~V}$ and a drop on the efficiency of $2 \%$ is observed.

The success of the rate capability test led to the longevity test start and it is in progress at the GIF++ facility. The longevity test aims to secure an optimal performance of the RE3-4/1 after an accummulated charge of $330 \mathrm{mC} / \mathrm{cm}^{2}$, equivalent to more than 10 years of HL-LHC operation. The extended RPC system is expected to enhance the performance and robustness of the muon trigger, corresponding with the coverage of the inner tracker in the extended $\eta$ region, opening the possibility of an independent trigger-based only on the tracker and RPCs hits. 

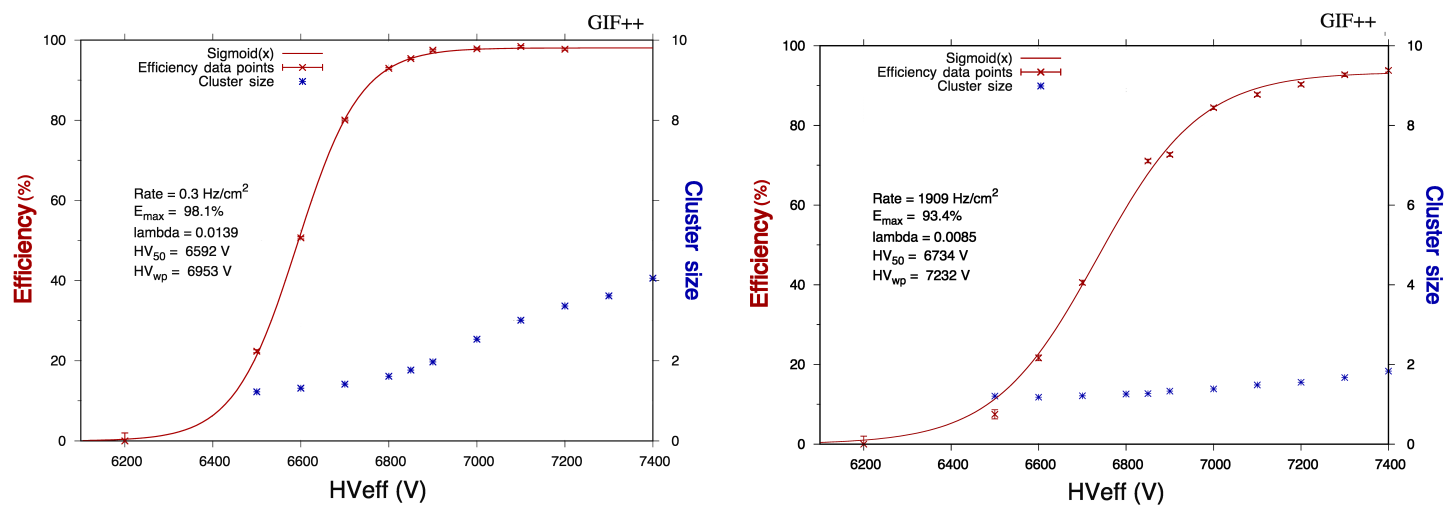

Figure 5. Efficiency and the average cluster size of a $1.4 \mathrm{~mm}$ double-gap RPC large size chamber as a function of the effective voltage, tested without gamma background (left) and under a gamma background rate of $1.91 \mathrm{kHz} / \mathrm{cm}^{2}$ (right).

\section{Sustainability of the current system}

The installed CMS RPCs are double-gap chambers with $2 \mathrm{~mm}$ gas width that operate in avalanche mode with a gas mixture of $95.2 \% \mathrm{C}_{2} \mathrm{H}_{2} \mathrm{~F}_{4}, 4.5 \% i \mathrm{C}_{4} \mathrm{H}_{10}$ and $0.3 \% \mathrm{SF}_{6}$ with a relative humidity of about $40 \%-50 \%$. The link system has the function to process and synchronize the signals coming from the RPC Front-End Boards (FEB) providing RPC hits with a time resolution of one bunch crossing (25 ns). During the HL-LHC operation, the expected conditions in terms of background and pile-up and the probable aging of the present detectors will make the muon identification and correct $p_{T}$ assignment a challenge for the muon system. In order to make certain the redundancy of the muon system in those conditions, the present RPC system has to be improved.

\subsection{Link Board upgrade for the current RPC system}

The Link System of the current RPC, composed of Link Boards (LB) and Control Boards (CB), is responsible for taking the signal from the Front End Boards in the chambers, synchronizing and compressing it, and sending it via the optical links to trigger processors. In order to secure the good operation of the RPC readout for the HL-LHC there are few weak points that need to be addressed:

- Operations: the CBs are connected into token ring logic configuration. Each ring consists of $12 \mathrm{CBs}$. One ring is responsible for half of a barrel wheel or half of two end-cap disks. If one $\mathrm{CB}$ fails then the entire ring does not work, leading to a loss of $6 \%$ of the system.

- Maintenance: it is a custom electronics, there are not enough LB/CB spares available, and we have to rely on unsupported ASICs.

- The rate of output optical signals is $1.6 \mathrm{~Gb} / \mathrm{s}$. This rate is slow for modern optical receiver interface and optical receivers might not be able to receive data at this rate in the future.

The upgrade of the link system will consist only of the replacement of the LBs and CBs, compatibles with the present Link Boxes, based on Xilinx 7 FPGAs. The new LBs will have the same dimensions, same back-plane connectors, and same pin assignment on the back-plane 
connectors as in the current system. Current technology allows us to absorb the functionality of most of the chips in the present link system into FPGAs. The ring configuration issue will be resolved by using a high-speed switchboard connecting each CB in parallel. The RPC signal sampling frequency will go from $40 \mathrm{MHz}$ (present system) to $640 \mathrm{MHz}$ (upgraded). Time resolution will be improved from $25 \mathrm{~ns}$ to $1.6 \mathrm{~ns}$, having an impact on muon trigger and offline reconstruction. Figure 6 shows that the time resolution achievable by using RPCs with the upgraded link system is about a factor two better than the one that the DT and CSC detectors can provide alone, overcoming the weaknesses described before.

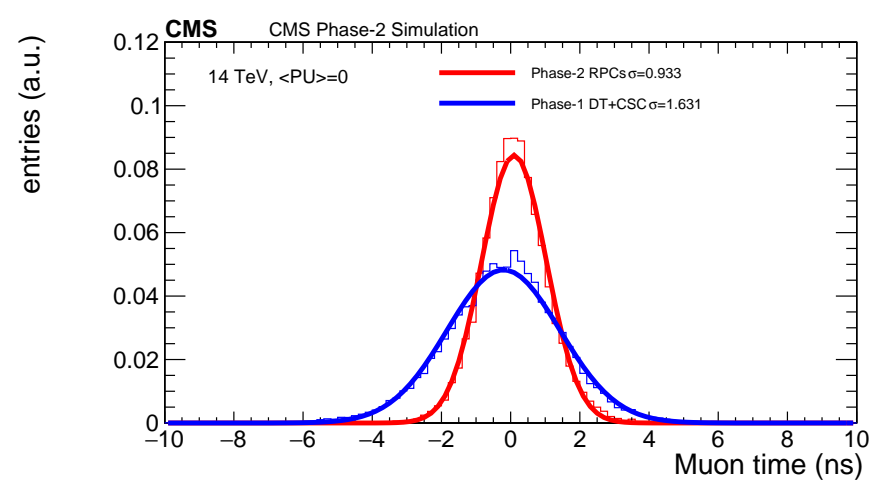

Figure 6. Time residuals between reconstructed and true times associated with a muon for the case when one uses the CSC and DT detectors alone (blue) and for the case of RPCs with the upgraded link system (red).

\subsection{Eco-gas studies}

The RPC gas mixture mainly consists of fluorinated gases (F-gas) with high Global Warming Potential (GWP) equal to 1307: $\mathrm{C}_{2} \mathrm{H}_{2} F_{4} 95.2 \% S F_{6} 0.3 \%-i C_{4} H_{10} 4.5 \%$. Where $C_{2} H_{2} F_{4}$ and $S F_{6}$ have the highest GWP and are crucial to ensure a stable detector performance in avalanche mode at the background conditions expected at HL-LHC (efficiency more than 95\% and time resolution $\equiv 1.5 \mathrm{~ns}$ with a rate capability of $\equiv 600 \mathrm{~Hz} / \mathrm{cm}^{2}$ ). Recent European regulations on environmental safety policy may lead to a restriction or banning on the use of fluorinated Greenhouse Gases $(\mathrm{GHG})^{*}$. This has motivated an extensive Research and Development (R\&D) program within the RPC community, two gases with low GWP: HFO-1234ze and $C F_{3} I$ have been studied previously [6] showing promising results. These two gasses have been tested using, in addition, $\mathrm{CO}_{2}$ in order to maintain the HV working point below $12 \mathrm{kV}$.

Figure 7 on the left shows that increasing the fraction of HFO-1234ze moves the working voltage to higher values and reduces the streamer probability ${ }^{1}$ at the working point, while on the right we see promising results with $50 \%$ of HFO, showing a $1.3 \mathrm{kV}$ shift in the HV working point. We have seen this with both current and iRPCs, with 50\% HFO, the efficiency is consistent with the one for the standard mixture with up to a $2 \mathrm{kV}$ shift of the $\mathrm{HV}$ working point.

${ }^{1}$ Defined as the fraction of events with an induced charge above $20 \mathrm{pC}$ at the working point. 

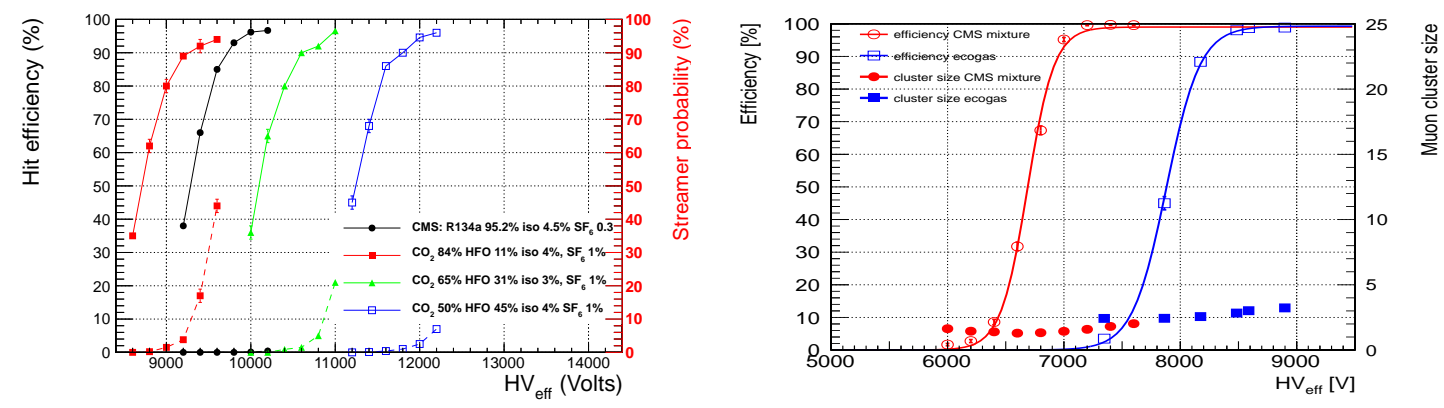

Figure 7. Hit Efficiency and streamer probability vs. effective voltage for different ecological gas mixtures on RPCs (left). Efficiency and Cluster size vs. effective voltage for the CMS gas mixture and an ecological gas mixture (right) used on iRPCs.

\section{Summary}

During the HL-LHC period, triggering on muons, muon identification and correct $p_{T}$ assignment will be a challenge for the muon system. In order to handle the high background conditions, the number of hits per track should be increased to strengthen the redundancy of the muon system to obtain a solid muon reconstruction. The required upgrade for the RPC system was presented. It implies to upgrade the link system and to extend the $\eta$ coverage with the installation of iRPCs in the innermost rings of stations 3 and 4 . The new link system will improve the precision in the timing readout allowing taking full advantage of the RPC intrinsic time resolution $(\sim 1.5 \mathrm{~ns})$, as it recovers the weaknesses and deterioration due to the aging of the present system. And an extensive $R \& D$ program is being performed in order to develop an iRPC that fulfills the CMS requirements for HL-LHC. In this report, we presented the validation of a double-gap large size prototype with a $1.4 \mathrm{~mm}$ electrode and $1.4 \mathrm{~mm}$ gap thickness working with a $95 \%$ efficiency at a rate of $2 \mathrm{kHz} / \mathrm{cm}^{2}$. We also presented promising results for the eco-gas increasing the amount of $\mathrm{HFO}$ over the $\mathrm{CO}_{2}$. Both upgrades are expected to be ready after the LHC Long Shutdown 3.

\section{References}

[1] CMS collaboration, The CMS muon project: Technical Design Report, CERN-LHCC-97-32 (1997).

[2] CMS collaboration, The Phase-2 Upgrade of the CMS Muon Detectors, CERN-LHCC-2017-012 (2017).

[3] G. Aielli et al., Improving the RPC rate capability, 2016 JINST 11 P07014 [arXiv: 1606. 03448].

[4] S.K. Park, M.H. Kang and K.S. Lee, RPC Gap Production and Performance for CMS RE4 Upgrade, 2014 JINST 9 C11009 [arXiv: 1408. 3720].

[5] K.S. Lee et al., Radiation tests of real-sized prototype RPCs for the Phase-2 Upgrade of the CMS Muon System, 2016 JINST 11 C08008 [arXiv: 1605. 00440]. 
[6] M. Abbrescia et al., Preliminary results of Resistive Plate Chambers operated with eco-friendly gas mixtures for application in the CMS experiment, 2016 JINST 11 C09018 [arXiv: 1605 . 08172].

[7] CMS collaboration, First results of CMS RPC performance at 13 TeV, 2016 JINST 11 C12003 [arXiv: 1605.09521].

[8] J. Fleury et al., Petiroc, a new front-end ASIC for time of flight application, in Proceedings of the 2013 IEEE Nuclear Science Symposium and Medical Imaging Conference (2013 NSS/MIC), Seoul Republic of Korea (2013) 